\title{
Photosensitive junctions based on UV-modified graphene and inkjet- printed organic molecules
}

Nekrasov, Nikita, Emelianov, Aleksei, Kireev, Dmitry, Omerović, Nejra, Bobrinetskiy, Ivan

Nikita Nekrasov, Aleksei Emelianov, Dmitry Kireev, Nejra Omerović, Ivan Bobrinetskiy, "Photosensitive junctions based on UV-modified graphene and inkjet-printed organic molecules," Proc. SPIE 11365, Organic Electronics and Photonics: Fundamentals and Devices II, 113650X (1 April 2020); doi: 10.1117/12.2560646

SPIE. Event: SPIE Photonics Europe, 2020, Online Only, France 


\title{
Photosensitive junctions based on UV-modified graphene and inkjet- printed organic molecules
}

\author{
Nikita Nekrasov ${ }^{* a}$, Aleksei Emelianov ${ }^{\mathrm{a}}$, Dmitry Kireev ${ }^{\mathrm{b}}$, \\ Nejra Omerovićc ${ }^{\mathrm{c}}$ Ivan Bobrinetskiy ${ }^{\mathrm{a}, \mathrm{c}}$ \\ aNational Research University of Electronic Technology, Moscow, Zelenograd, 124498, Russia; \\ ${ }^{b}$ Department of Electrical and Computer Engineering, The University of Texas at Austin, Austin, \\ TX, 78758, USA; 'BioSense Institute-Research Institute for Information Technologies in \\ Biosystems, University of Novi Sad, Dr Zorana Djindjica 1a, 21000 Novi Sad, Serbia
}

\begin{abstract}
In this work, we report a novel method of mask-less doping of graphene channel in field-effect transistor configuration by local inkjet printing of organic semiconducting molecules. Graphene-based transistor was fabricated via large-scale technology, allowing for upscaling electronic device fabrication and lowering the device cost. The altering of functionalization of graphene was performed through local inkjet printing of semiconducting molecules: N,N'-Dihexyl-

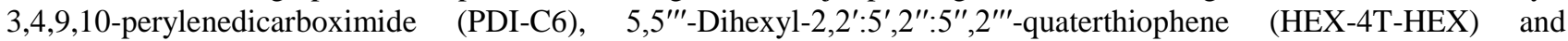
polyalanine (PANI). We found the effect of UV treatment on fabrication of graphene/organic junctions because of change in graphene hydrophobic properties. We demonstrated the high resolution (about $50 \mu \mathrm{m}$ ) and accurate printing of organic ink on UV treated chemical vapor deposited (CVD) graphene. The PANI/graphene junction demonstrate more stable photoresponse characteristic for $470 \mathrm{~nm}$ diode illumination. The characteristics of PDI/graphene junction demonstrate the saturation for high diode power because of organic crystals degradation. The photoresponse of $1 \mathrm{~mA} / \mathrm{W}$ was demonstrated for PANI/graphene junction at $0.3 \mathrm{~V}$ bias voltage. The developed method opens the way for local functionalization of on-chip array of graphene by inkjet printing of different semiconducting organic molecules for photonics and electronics application.
\end{abstract}

Keywords: CVD graphene; field-effect transistor; junction; UV functionalization; semiconducting organic molecules; inkjet printing; photoresponse

\section{INTRODUCTION}

Graphene is one of the most promising materials for advanced electronic applications, due to its extraordinary chemical and physical properties ${ }^{1,2}$. So far, the graphene application in electronics is still challenging, perspectives in photonics and optoelectronics are more defined ${ }^{3,4}$. Different optoelectronic devices based on graphene, such as transparent electrodes $^{5}$, photovoltaic devices ${ }^{6}$, light emitting diodes ${ }^{7}$, photodetector $^{8}$, ultrafast lasers ${ }^{9}$ and $\mathrm{THz}$ detectors ${ }^{10}$ were demonstrated. The major challenge in graphene optoelectronics is in its planar nature that poses the way for in-plane integrated devices. Thus, the development of planar functional junction can provide continuous, single-atom thick, inplane integrated circuits. The production of atomic contacts of different materials (hetero-/homojunctions) is still a challenging task for $2 \mathrm{D}$ materials technology ${ }^{11}$. One of the main problems is still the graphene surface that is hydrophobic and can contain different contaminants that prevent good adsorption and charge transfer in graphene junctions with other materials. Proper functionalization of graphene is needed before assembling of advanced devices.

Recently it was demonstrated that ultraviolet (UV) irradiation is not only a useful method to eliminate the polymethyl methacrylate (PMMA) residues, but it can also be exploited to control the surface functionality more efficiently ${ }^{12,13}$. The UV-assisted oxidation can affect the charge carriers concentration in graphene ${ }^{14}$. The doping is caused by the formation of carboxyl and carbonyl groups, formed at the graphene's surface in the presence of water and UV-light. Moreover, the reversible wettability transition between hydrophobic and hydrophilic graphene under UV irradiation has been demonstrated ${ }^{15}$. Thus, proper control of UV light irradiation can provide both cleaning, doping and surface energy modification of graphene in task of junction development with other materials.

*vkn@nanotube.ru; phone +7 49972089 22; www.nanotube.ru 
This paper reports on the maskless method of hybrid photosensitive junction fabrication in a graphene field-effect transistors (GFET) based on the inkjet printing of semiconducting organic inks. While graphene serves as a conductive channel, the non-covalent functionalization with semiconducting organic molecules alters the optical sensitivity in normal conditions to the visible wavelengths. We demonstrated the two-step technique of graphene-channel functionalization by UV processing and graphene/organic junction formation by local printing of organic inks. The electrical and optical characterization of modified devices were investigated. The mechanisms of optical irradiation on the devices are discussed.

\section{METHODOLOGY}

\subsection{Graphene-field effect-transistors (GFET) fabrication}

GFET was produced from CVD graphene by transferring to $\mathrm{SiO}_{2}$ substrate and electrode patterning by method described elsewhere ${ }^{16}$. Briefly CVD-grown graphene was wet-transferred onto $300 \mathrm{~nm} \mathrm{SiO}{ }_{2}$ on highly doped $\mathrm{Si}\left(\mathrm{p}^{++}\right)$substrate. The photolithography was applied to pattern $\mathrm{Au} / \mathrm{Ti}$ electrodes on the plasma patterned $20 \times 20 \mu \mathrm{m}$ graphene channels. The prepared GFETs chip was washed $5 \mathrm{~min}$ in acetone and $5 \mathrm{~min}$ in isopropyl alcohol (IPA). After it was washed under DI water and dried in normal conditions. The graphene was UV processed in ambient conditions and in humid environment.

\subsection{UV processing}

Surface of GFETs was UV treated in the air to remove the organic residuals and activate the carbon bonds. To process GFETs, we use two types of UV sources. For in situ experiments UV treatment was performed under 395nm UV light emitted diode (LED) with focusing lens at $1 \mathrm{~cm}$ distance from substrate during $5 \mathrm{~min}$. For routing UV processing before inkjet printing a low-pressure mercury lamp Svetolit-50 (LIT, Russia) with $20 \mathrm{~W}$ irradiation power at $191 \mathrm{~nm}$ wavelength was used for $10 \mathrm{~min}$.

\subsection{Inkjet printing}

We used the following semiconducting organic molecules $N, N^{\prime}$-Dihexyl-3,4,9,10-perylenedicarboximide (PDI-C6), 5,5'"-Dihexyl-2,2':5',2":5",2'"-quaterthiophene (HEX-4T-HEX) (Sigma Aldrich, USA), and polymer polyalanine (PANI) (Sigma Aldrich, USA) for ink preparation. For ultrasound treatment of the ink, the ultrasound bath Bandelin Sonorex was used for $30 \mathrm{~min}$. The top layer of the solution was taken for inkjet cartridge filling. The ink was filtered using a filter with pore size of $200 \mathrm{~nm}$ (Chromafil CA-20/25, cellulose acetate) before loading to the cartridge.

For inkjet printing, Dimatix Materials Printer DMP-3000 (Fujifilm, Tokyo, Japan) inkjet printer was used. The printer operates via piezoelectric jetting cartridges with $21.5 \mu \mathrm{m}$ nozzle diameters. In order to obtain a controllable process, basic printing parameters, such as waveform, frequency, jetting voltage, and drop spacing, were adjusted. For lowviscous ink $(<2 \mathrm{cP})$, the waveform of pulse supplied on piezoelectric nozzles is the main parameter to control the formation of the stable droplets ${ }^{17}$. To ensure stable conditions during the process and proper layer formation, the substrate and the cartridge worked at room temperature. All the experiments with organic inkjet printing were performed in cleanroom facilities.

\subsection{Graphene FET analysis}

Optical characterization was performed on optical profiler HRM-300 (Huvitz BD, Dongan, Republic of Korea) with 5×$50 \times$ objectives). Raman measurements are performed at $532 \mathrm{~nm}$ excitation wavelength with $1 \mathrm{~mW}$ power using a microRaman system Centaur HR (Nanoscan Technology, Russia). The laser is focused to $1.5 \mu \mathrm{m}$ spot through an x100 Olympus objective. Atomic-force microscopy (AFM) using Solver-Pro (NT-MDT, Russia) with standard silicon cantilevers NSG03 series in semi-contact mode with $\mathrm{f}_{\text {res }}=90 \mathrm{kHz}$ is performed to characterize the topography of the graphene surface. Electrical characteristics are measured via semiconductor device analyzers Keithley 4200 and IPPP1/5 (MNIPI, Belarus). Transfer I-V curves are measured using backgate $\left(\mathrm{p}^{++} \mathrm{Si}\right)$ or liquid gate $(\mathrm{Ag} / \mathrm{AgCl})$ electrode.

To evaluate the photocurrent response and conduct the photocurrent measurements, $470 \mathrm{~nm}$ LED was used (Thorlabs, Newton, NJ, USA). An LED was placed at a distance of $10 \mathrm{~cm}$ above the structures. The experiments were performed in the room light and in the light-protected box with and without LED illumination. 


\section{RESULTS}

\subsection{Organic ink preparation}

The solutions with organic molecules were prepared with different solvents, such as n-methyl-2-pyrolidone (NMP), toluene, chlorobenzene (CB), isopropyl alcohol (IPA) in different concentrations. The optimization process needed to fit both demands for inkjet printing: (1) surface tension close to $\mathrm{Si} / \mathrm{SO}_{2}$ substrate ${ }^{18}(36 \mathrm{mN} / \mathrm{m})$ and (2) high enough viscosity to provide stable droplets formation $(>1 \mathrm{cP})$. Finally, we found optimal solutions for each molecules that provide stable ink formation (Table 1).

Table 1. Parameters of organic ink preparation.

\begin{tabular}{|l|l|l|l|}
\hline \multicolumn{1}{|c|}{ Parameter } & \multicolumn{1}{c|}{ PANI } & \multicolumn{1}{c|}{ HEX-4T-HEX } & \multicolumn{1}{c|}{ PDI-C6 } \\
\hline $\begin{array}{l}\text { Molecules(mg):solvent } \\
\text { 1(ml):solvent 2(ml) }\end{array}$ & $\begin{array}{l}1,4 \mathrm{mg}: 6,7 \mathrm{ml}(\mathrm{NMP}): 1,7 \\
\mathrm{ml}(\mathrm{IPA})(80 / 20 \%)\end{array}$ & $\begin{array}{l}1,2 \mathrm{mg}: 3,6 \mathrm{ml}(\mathrm{CB}): 3,4 \\
\mathrm{ml}(\mathrm{IPA})(50 / 50 \%)\end{array}$ & $\begin{array}{l}0,7 \mathrm{mg}: 3 \mathrm{ml} \text { (toluene):3 ml } \\
\text { (IPA) (50/50\%) }\end{array}$ \\
\hline Ultrasound & \multicolumn{3}{|c|}{$30 \mathrm{~min}$} \\
\hline
\end{tabular}

PANI is known as admixture for some solutions for viscosity increasing, because itis a long chain molecule, so the solution with these molecules is viscous enough in NMP solution. Other dyes are oligomers and they are polar molecules, so NMP is not preferable, because it is polar solvent (dipole moment $4.12 \mathrm{D}$ ). The inks of $0.7 \mathrm{mg}$ of PDI-C6 were prepared in $6 \mathrm{~mL}$ of toluene/IPA $(50 / 50 \%)$ solution. Toluene (dipole moment $0.36 \mathrm{D})$ was used as a less polar solvent for PDI. As far as the viscosity of toluene is small $(0.56 \mathrm{cP})$, we added IPA $(2.04 \mathrm{cP})$ to prepare the ink. Chlorobenzene $(1.59 \mathrm{D})$ and toluene $(0.36 \mathrm{D})$ are less polar solvents, so they are good candidates for dissolving. But the viscosity of $\mathrm{CB}$ and toluene are small ( 0.753 and $0.56 \mathrm{cP}$ respectively). To increase viscosity IPA was added in $50 \%$ of solution. We found HEX-4T-HEX formed stable solution, but PDI ink still had sediments. After preparations, ink was loaded to the cartridge. When one material was printed, the cartridge was cleaned by IPA, dried and then another material was loaded.

\subsection{UV processing of graphene channel}

First, we processed the graphene FETs in facilities with inkjet printer to see in situ the effect of UV on printing process. We used the hand-held UV diode (Figure 1a). After UV processing we put the GFET chip under objective of optical microscope and made a droplet on the surface to see its evaporation on-line. The speed of evaporation was controlled by heating through the microscope lamp power. During evaporation we observed difference in water droplet behaviour on GFET before and after UV treatment (fig 1). We found that before UV processing no droplet remained on graphene channel. Water drop was just held between gold electrodes by capillary forces. On the contrary the UV processed channel held the residual droplets drying on its surface.

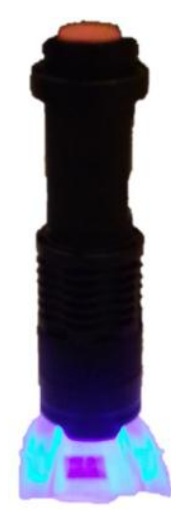

$\mathrm{a}$
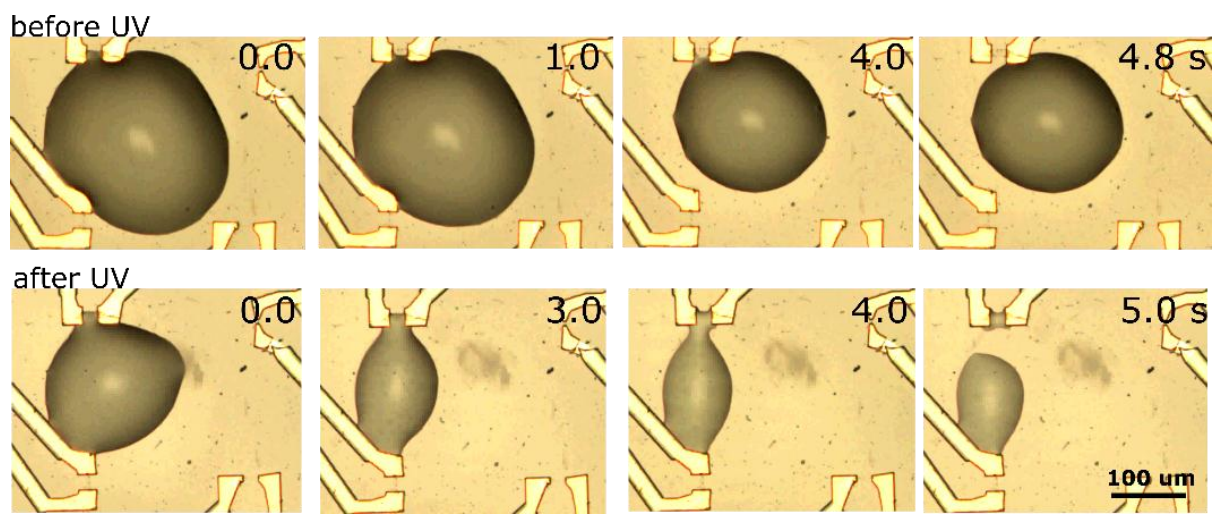

b

Figure 1. The UV processing of graphene FET channel. The UV LED setup with GFET chip (a). The set of optical images of water microdroplet drying on graphene channel between gold electrodes before and after UV processing in different time periods (b). The time data are given in seconds. Scalebar is $100 \mu \mathrm{m}$. 
It is known that pristine graphene is hydrophobic and that it can be reversibly changed to the hydrophilic state using UV irradiation ${ }^{15}$. Different mechanisms responding to UV-irradiation can contribute to the changes in the properties of single-layer and few-layer graphene ${ }^{13}$. Formation of a carboxyl/carbonyl group requires breaking of a $\mathrm{C}$-C bond (energy around $3.7 \mathrm{eV}$ ). The mercury lamp with a mean peak at $191 \mathrm{~nm}$ provides energy of $6.5 \mathrm{eV}$, which should be enough to break the bond in a short time. While the formation of the holes leads to breaking of a $\mathrm{C}-\mathrm{C}$ bond, the valence of the atoms is compensated by the formation of the $\mathrm{C}-\mathrm{O}$ bonds. Under $\mathrm{UV}$ irradiation $\mathrm{H}_{2} \mathrm{O}$ molecules are split into hydrogen and hydroxyl radicals, which are then captured by the graphene surface through chemical binding in an ambient environment under UV irradiation. The dissociative adsorption of $\mathrm{H}_{2} \mathrm{O}$ molecules induces the wettability transition in graphene from hydrophobic to hydrophilic. Thus, the changing the power of UV source one can process graphene from an hour ${ }^{15}$ to several minutes ${ }^{13}$ to achieve desired functionality of graphene surface.

For printed organic ink we did not observe the presents of printed pattern on untreated graphene. The printing process was successful only after GFET was processed under low-pressure mercury lamp. This observation supports the model of increasing hydrophilic properties of graphene after UV processing, that lead to the lowering the surface tension.

\subsection{Photoresponse in graphene/organic junction under laser irradiation}

Because of high luminescence properties in visible range, we can easily observe the organic molecules monolayers printed on graphene channel (figure 2a, c). The printed dot of about $50 \mu \mathrm{m}$ is larger than the channel width of $20 \mu \mathrm{m}$. Nevertheless the optimization of organic monomers inks the still "coffee" ring is observed (figure 2c). The organic monomers are deposited in crystalline forma in all area of the drop. The monolayers of PANI molecules are transparent in visible range (figure $2 b$ ) and it spreads uniformly other the drop that proves the high quality of prepared solution. We used atomic force microscopy (AFM) to characterize the graphene/organic molecules junction. The film of Hex-4T-Hex and PDI-C6 consist of organic crystals forming molecular island-like layer and some aggregates (figure 2d, f) in agreement with the deposition of the ink on the bare graphene ${ }^{17}$. PANI forms uniform film covering mostly UV treated graphene channel (figure 2e).
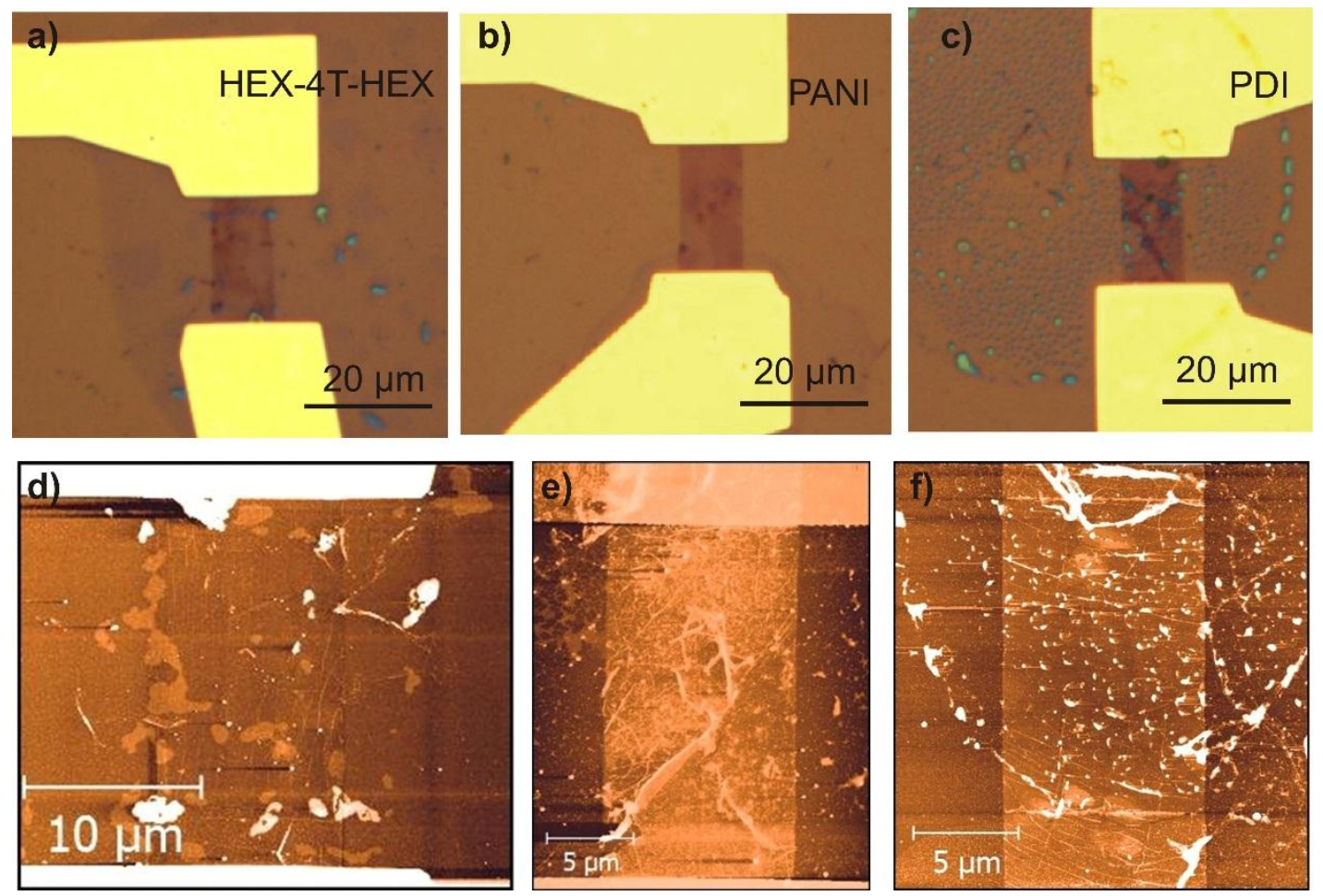

Figure 2. Optical image of a single drop of HEX-4T-HEX (a), PANI (b), PDI (c) inks printed on the graphene channels (scalebar is $20 \mu \mathrm{m}$ ). AFM images of the graphene channel of FET modified by HEX-4T-HEX (d), PANI (e), PDI (f) printed inks after drying (scalebar is $10 \mu \mathrm{m}$ for (d) and $5 \mu \mathrm{m}$ for (e) and (f)).

To investigate the photoresponsivity of the graphene/organic junctions, we performed characterization under LED light. The devices were illuminated with $470 \mathrm{~nm}$ LED with power density $20 \mu \mathrm{W} / \mathrm{cm}^{2}$. In Figure 3, we show that graphene 
junctions with different organic molecules has different respond when irradiated with a $470 \mathrm{~nm}$ light source. While for Hex-4T-Hex we observed very low photocurrent $\left(I_{\mathrm{ph}}\right)$ (figure 3a) on the level of measurement set-up error, the junctions with PANI and CDI provide rather strong photoresponsivity (figure $3 \mathrm{~b}$ and $3 \mathrm{c}$, respectively). Moreover, while for PANI/graphene junction there is no noticeable current for up to $50 \mathrm{mV}$ bias voltage, the photocurrent in PDI/graphene junction has linear dependence versus bias voltage up to $100 \mathrm{mV}$. The PDI molecules has structure similar to graphen providing $\pi-\pi$ stacking and low barrier between molecules and graphene. Polymer structure is more complicated and can result in higher barrier energy for charde transfer from PANI to graphene. This photogenerated carrier transport phenomenon demonstrates photoinduction modulating the graphene channel carrier concentration without gate control.
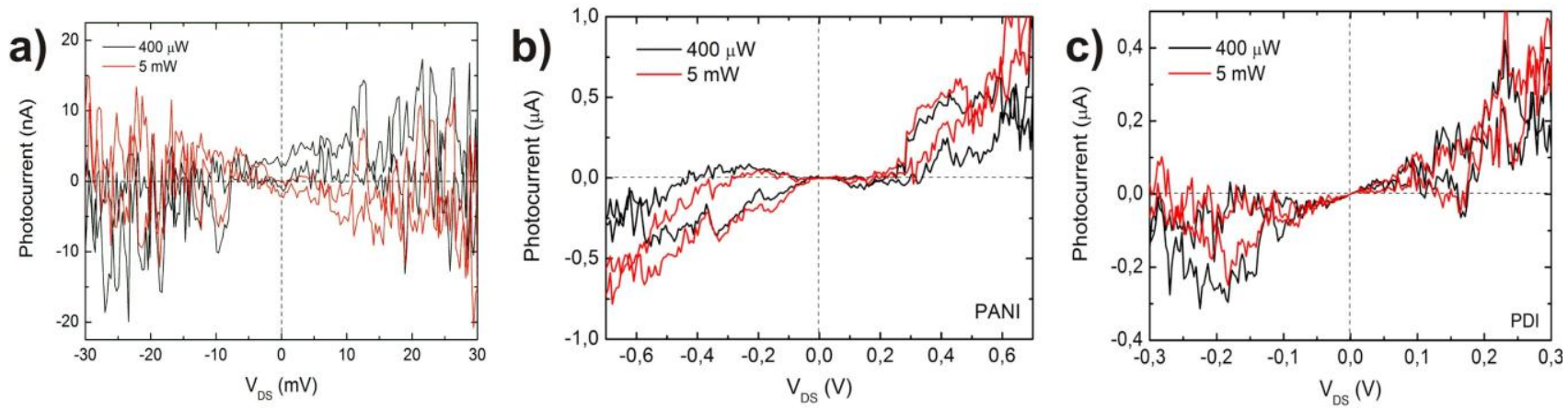

Figure 3. Photocurrent as a function of source-drain voltage measured for $470 \mathrm{~nm}$ diode light illumination for HEX-4THEX (a), PANI/graphene (b) and PDI/graphene junctions (c) for two different irradiation powers.

The photoresponse in graphene/organic interface can have either photovoltaic ${ }^{19}$ or photothermoelectric nature ${ }^{20}$. Organic molecules deposited onto graphene can dope or accept electrons from the channel converting it to an $n$-type region or $p$ type, respectively. On the other hand, the significant charge transfer from the molecules occurred only under light irradiation, while there was no real doping observed for modified graphene transistor in the dark, which indicates that the photovoltaic effect takes precedence over the thermoelectric effect in this configuration with a low bias regime ${ }^{21}$.

We suggest the Hex-4T-Hex/graphene junction cannot provide good efficiency in electron transfer because of low concentration and large monolayer islands observed by AFM (figure 2d). Very low number of generated charge carries from Hex-4T-Hex can reach on of the electrode to generate weak photcurrent. While PANI and PDI covers almost all the channel of graphene FET. For the structure with PDI molecules the photocurrent was low. The current displays noise increasing with bias voltage as well as with irradiation power increasing. Moreover the photocurrent for PDI/graphene junctions is decreasing with high irradiation power. We suggest the increasing of molecules mobility under high power that lead to characteristics degradation. The photocurrent of pure graphene/organic junction is typically very low because weak epitaxial relations between graphene and organic molecules. Thus, some buffer layers like fullerene ${ }^{22}$ is needed. For PANI/graphene junction the photocurrent was high upon diode illumination $\left(\sim 0.5 \mu \mathrm{A}\right.$ at $\mathrm{V}_{\mathrm{DS}}=0.6 \mathrm{~V}$ and $\left.\mathrm{P}=0.4 \mathrm{~mW}\right)$ and increases with irradiation power that suggest the effective charge transfer because of uniform polymer coverage. The maximum responsivity $(R)$ for $470 \mathrm{~nm}$ LED was calculated as $R=I_{\mathrm{ph}} / P$ (where $P$ - is an incident light power), which is equal to $1 \mathrm{~mA} / \mathrm{W}$ was demonstrated for PANI/graphene junction at $0.3 \mathrm{~V}$ bias voltage.

\section{CONCLUSIONS}

In conclusion, we have developed robust and scalable technology for the functionalization of arrays of graphene fieldeffect transistors by inkjet printing of organic molecules monolayers. We prepared the semiconducting organic ink and studied the deposition of organic molecules onto the graphene surface. We found the effect of UV treatment on fabrication of graphene/organic junctions because of change in graphene hydrophobic properties by water species photochemical adsorption. We demonstrated the high resolution (about $50 \mu \mathrm{m}$ ) and accurate printing of organic ink on UV treated CVD graphene. This technology is very flexible and can provide the modification of an array of GFET on the same substrate with different organic molecules that can be tuned for specific wavelengths. The increase of photocurrent functionalized graphene with N,N'-Dihexyl-3,4,9,10-perylenedicarboximide and polyalanine molecules monolayers were shown. The junction is sensitive to $470 \mathrm{LED}$ light with a photoresponse up to $1 \mathrm{~mA} / \mathrm{W}$ at $0.3 \mathrm{~V}$ bias voltage and $400 \mu \mathrm{W}$ light intensity for PANI/graphene junction. We suggested that the photovoltaic effect is responsible for photocurrent generation in a junction created by light-induced doping of graphene from organic molecules. 


\section{ACKNOWLEDGEMENTS}

The research was supported by Russian Science Foundation under grant number 19-19-00401

\section{REFERENCES}

[1] Novoselov, K. S., Geim A. K., Morozov S. V., Jiang D., Zhang Y., Dubonos S. V., Grigorieva I. V., Firsov A. A., "Electric field effect in atomically thin carbon films," Science 306, 666 (2004).

[2] Geim, A. K., Novoselov, K. S., "The rise of graphene," Nature Mater. 6, 183 (2007).

[3] Ferrari, A. C., Bonaccorso F., Falko V., Novoselov K. S., Roche S., Bøggild P., Van Wees B. J., "Science and technology roadmap for graphene, related two-dimensional crystals, and hybrid systems," Nanoscale 7, 4598 (2015).

[4] Bonaccorso, F., Sun, Z., Hasan, T., Ferrari, A. C., "Graphene photonics and optoelectronics," Nature photonics 4(9), 611 (2010).

[5] Liu, Z., Parvez, K., Li, R., Dong, R., Feng, X., Müllen, K., "Transparent conductive electrodes from graphene/PEDOT: PSS hybrid inks for ultrathin organic photodetectors," Advanced Materials 27(4), 669 (2015).

[6] Liu, Z., Lau, S. P., \& Yan, F., "Functionalized graphene and other two-dimensional materials for photovoltaic devices: device design and processing," Chemical Society Reviews 44(15), 5638 (2015).

[7] Zhang, Z., Du, J., Zhang, D., Sun, H., Yin, L., Ma, L., et al., "Rosin-enabled ultraclean and damage-free transfer of graphene for large-area flexible organic light-emitting diodes," Nature communications 8, 14560 (2017).

[8] Kang, P., Wang, M. C., Knapp, P. M., \& Nam, S., "Crumpled Graphene Photodetector with Enhanced, Strain- Tunable, and Wavelength- Selective Photoresponsivity," Advanced Materials, 28(23), 4639 (2016).

[9] Wan, H., Cai, W., Wang, F., Jiang, S., Xu, S., Liu, J., "High-quality monolayer graphene for bulk laser modelocking near $2 \mu \mathrm{m}$," Optical and Quantum Electronics 48(1), 11 (2016).

[10] Bandurin, D. A., Gayduchenko, I., Cao, Y., Moskotin, M., Principi, A., Grigorieva, I. V., Goltsman G. N., Fedorov G.E., Svintsov, D., "Dual origin of room temperature sub-terahertz photoresponse in graphene field effect transistors, “ Applied Physics Letters 112(14), 141101 (2018).

[11] Solís-Fernández, P., Bissett, M., \& Ago, H., "Synthesis, structure and applications of graphene-based 2D heterostructure," Chemical Society Reviews 46(15), 4572 (2017).

[12] Kim, J. H., Haidari, M. M., Choi, J. S., Kim, H., Yu, Y. J., Park, J., "Facile Dry Surface Cleaning of Graphene by UV Treatment," Journal of the Korean Physical Society 72(9), 1045-1051 (2018).

[13] Emelianov, A. V., Kireev, D., Levin, D. D., Bobrinetskiy, I. I., "The effect of ultraviolet light on structural properties of exfoliated and CVD graphene," Applied Physics Letters 109(17), 173101 (2016).

[14] Imamura, G., Saiki, K., "Modification of graphene/SiO2 interface by UV-irradiation: effect on electrical characteristics," ACS applied materials \& interfaces 7(4), 2439-2443 (2015).

[15] Zhang, X., Wan, S., Pu, J., Wang, L. Liu, X., "Highly hydrophobic and adhesive performance of graphene films," J. Mater. Chem. 21, 12251-12258 (2011).

[16] Kireev, D., Brambach, M., Seyock, S., Maybeck, V., Fu, W., Wolfrum, B., Offenhäusser, A., "Graphene transistors for interfacing with cells: towards a deeper understanding of liquid gating and sensitivity," Scientific reports 7(1), 6658 (2017).

[17] Nekrasov, N., Kireev, D., Omerović, N., Emelianov, A., Bobrinetskiy, I., "Photo-Induced Doping in a Graphene Field-Effect Transistor with Inkjet-Printed Organic Semiconducting Molecules," Nanomaterials 9, 1753 (2019).

[18] Hu, G., Kang, J., Ng, L.W., Zhu, X., Howe, R.C., Jones, C.G., Hersam, M.C. and Hasan, T., "Functional inks and printing of two-dimensional materials," Chemical Society Reviews 47(9), 3265-3300 (2018).

[19] Kim, J.B., Li, J., Choi, Y., Whang, D., Hwang, E., Cho, J.H., "Photosensitive graphene P-N junction transistors and ternary inverters," ACS Appl. Mater. Inter., 10, 12897-12903 (2018).

[20] Wang, S., Sekine, Y., Suzuki, S., Maeda, F., Hibino, H., "Photocurrent generation of a single-gate graphene p-n junction fabricated by interfacial modification," Nanotechnology, 26, 385203, (2015).

[21] Freitag, M., Low, T., Xia, F., Avouris, P., "Photoconductivity of biased graphene," Nat. Photonics 7, 53 (2013).

[22] Han, J., Wang, J., Yang, M., Kong, X., Chen, X., Huang, Z., Guo, H., Gou, J., Tao, S., Liu, Z., Wu, Z., Jiang, Y., Wrang, X., "Graphene/organic semiconductor heterojunction phototransistors with broadband and bi- directional photoresponse, " Advanced materials 30(49), 1804020 (2018). 